of the Department of Agriculture on their standardization, was attracting considerable attention among pharmacologists. Much interest was expressed in the work of the Council on Pharmacy and Chemistry; an exceptionally well-informed pharmacologist expressed the opinion that the work of the Council is driving some of the American frauds to England. as they are advertising in the British journals. The British physicians are asking what these widely advertised American drugs are. Some of those mentioned have been exposed by the Council on Tharmacy and Chemistry and the discussion of them in the Propaganda for Reform in Proprietary Medicines was read with much interest. It is claimed that the leading Fnglish manufacturers who seek the patronage of the medical profession refuse to make secret proprietary preparations for exploitation to either the profession or the public.

Two interesting articles, dealing to a greater or less extent with pharmacologic problems, have recently appeared in English journals. One, an address by Sir Lauder Brunton, shows how greatly the resources of the physician have been increased within the memory of those still very active in the profession.

Speaking of the conditions which prevailed when he was a hospital interne forty years ago, Brunton says: "We had no antisepsis." "I have heard people speak of the folly of an antiseptic spray during operations, but I do not think such people could have had any idea of the condition of operating theaters when the antiseptic method was introduced." "The medicines at our disposal for the treatment of diseases were few in comparison with those we now have, especially in those such as are useful in the treatment of sleeplessness and the relief of pain. For example, when one of my fellow residents had typhus fever we had no remedies to combat the distressing sleeplessness except opium combined, as the fever was high, with tartar emetic, for cannabis and hyoscyamus had proved inefficient, and $I$ well remember the anxiety with which we watched to see whether a 'new remedy' for sleeplessness, viz., bromid of potassium, would produce the desired effect. Now we have chloral, sulphonal, trional, veronal, paraldehyd and many others." Since the time of which Brunton wrote such drugs as the antiseptics, the antipyretics, cocain, chloral, theobromin, sulphonal, eserin, the therapeutic value of the suprarenal and thyroid, and many others have been discovered; the most pronounced therapeutic nihilist will not deny that many of these are immense gains.

Cushny, pointing out the value of experiments on animals, has recently traced the source of most of these new drugs. ${ }^{1}$ The following sentences are significant. "No soporific has been introduced in the last forty years, except by means of animal experiments." "No local anesthetic has been discovered, except by animal experiment." He found the same to be true for the antipyretics, eserin, strophanthus, all of the vascular dilators, suprarenal extract, caffein and theobromin, hexamethylenamin ("urotropin"), heroin and many others. "Excluding the local antiseptics which are used not to act on the patient, but on his parasites, he found that in the last forty years the only drug of even mediocre importance introduced by other methods was pilocarpin, which was introduced from its being used by the South Americans as a sudorifie."

\section{Treatment of Burns.}

Denver, Oct. 24, 1907.

To the Editor:-In reading the article in THE Journal, September 7, page 863, on "Treatment of Burns," my attention was called to the number of picric acid formulas recommended. I was also reminded of $\mathrm{my}$ own personal experience with the use of this compound after a severe bromin burn and the excruciating pain it caused me to suffer. After several dressings of picric acid solution on gauze strips, with as many periods of torture thereby induced, I decided to use some agent which was both antiseptic and anesthetic. Carbolic acid was, of course, one of the first to be thought of, but discarded be. cause of its tendency to produce gangrene. It was then that liquor cresolis comp. was tried with the most gratifying results. Since then, some twelve years past, I have had many

1. Cushny : Brit. Med. Jour., 1907, i, 1195. occasions to employ it, and whether the burn was due to acids, steam, hot water or flame, the immediate relief of pain followed its application.

Having reported its use some years ago, I had expected that others would probably try it and that we would have further reports, bat so far I have failed to see them recorded. While most burns are sterile by reason of the destructive agent, yet in burns involving any considerable surface, and produced through the wearing apparel, it is quite reasonable to expect some degree of infection. To elean the surface of these burns, and also render it both sterile and anesthetic, the use of but one solution has saved me much time and the patients considerable suffering. As the aqueous solution of liquor cresolis comp. is saponaceons, I have been using a $1 / 2$ to 1 per cent. warm solution to bathe the injured part and wash away the dobris; by the time this is accomplished the anesthesia is established. The blebs are then punctured and the serum expressed, after which gauze strips, spread with a 1 per cent. ointment of liquor cresolis comp. in petroleum, are applied over the entire burned surface, then covered with cotton and bandaged. This dressing may remain for four or five days, when it can be changed for any form which seems best to the attending surgeon. I have seldom found it necessary to use opiates after this form of dressing. Dr. Roosevelt has been using this compound recently after bromin burns. He finds that if used immediately and before there is much tissue destruction, it quickly neutralizes the chemical. He uses from a 25 per cent. to a full strength solution, finally washing this off when the pain is relieved and the skin but little damaged.

\section{E. Texnaxt.}

\section{A New Disease?-zutointoxication?} HoMer, NeB., Oct. 30, 1907.

To the Editor:-It was with much interest $I$ read the article of Dr. W. E. Clark of Frederick, S. D., in The Journal, Oct. 12,1907, p. 1295, describing a number of cases of a new disease which he had seen. I saw my first case, similar to those he described, about the middle of July, and since that time I have seen a great many, but none for the past month.

The majority of cases were in young adults. Neither milk nor any excess in diet could be held accountable. The symptoms were the same as described by Dr. Clark. In one case, in which the patient was wildly delirious, there was a temperature of $104 \mathrm{~F}$. In all the cases, when the headache subsided, the patients felt very comfortable, as it was the most painful feature of their illness. The bowels usually became loose, several free movements during the day. The appetite suddenly returned and, with the exception of a feeling of weakness, they felt well. In every case, the loss of flesh was marked, as none was sick a week and all lost from 10 to 15 pounds each. The headache lasted from 36 hours to 4 days. I finally concluded that it was an autointoxication through the intestinal canal, with a markedly torpid condition of the liver. I gave broken doses of calomel, followed by a saline cathartic, until there was a free action of the bowels; diuretics, such as potassium acetate; granular effervescent sodium phosphate as a hepatic stimulant; ice cap or cold water to the head; hot footbaths and confinement in a darkened room. Opiates had little effect, unless large doses of morphin were given. There were no fatalities.

Nina R. Smrti.

\section{Therapeutics Not Necessarily Drug Giving.}

$$
\text { Fort Wayne, Ind., Oct. } 31,1907 .
$$

To the Editor:-Addresses on pharmacology in THE JOURNaL complain of the ignorance and indifference of the medical profession to this branch of medical science. But are not the medical colleges and the American Medical Association responsible for these things? The colleges have chairs of "materia medica and therapeutics," and the American Medical Association has a Section on Pharmacology and Therapeutics. Why therapeutics? There are several therapies, such as surgery, besides pharmacotherapy. There is no reason why drugs and therapeuties should be so combined. It makes the public believe that treatment and drugging are the same thing. A judge has defined the practice of medicine as the prescribing of drugs; 